RUNNING HEAD: ONLINE CBT FOR ADOLESCENT ANXIETY

\title{
A randomized controlled trial of online versus clinic-based CBT for adolescent anxiety
}

\author{
Susan H. Spence (PhD, MBA) ${ }^{1}$ \\ Caroline L. Donovan $(\mathrm{PhD})^{1}$ \\ Sonja March $(\mathrm{PhD})^{1}$ \\ Amanda Gamble (PhD) ${ }^{2}$ \\ Renee E. Anderson (BPsychSc Hons) ${ }^{3}$ \\ Samantha Prosser (BPsychSc Hons) ${ }^{1}$ \\ Justin Kenardy $(\mathrm{PhD})^{34}$
}

June 5, 2010

\author{
Author Note: \\ ${ }^{1}$ School of Psychology, Griffith University \\ ${ }^{2}$ Department of Psychology, Macquarie University \\ ${ }^{3}$ School of Psychology, University of Queensland \\ ${ }^{4}$ Centre of National Research on Disability and Rehabilitation, School of Medicine \\ University of Queensland
}

Corresponding author: Caroline L Donovan, Griffith University, QLD, Australia TEL +61 73735 3401, FAX +61 737353388

Email: c.donovan@griffith.edu.au 


\begin{abstract}
Objective: The study examined the relative efficacy of clinician-assisted, online (NET) delivery of cognitive behavior therapy compared to clinic (CLIN) delivery of the same program and a wait list control (WLC) in the treatment of anxiety disorders in adolescents. Method: Participants included 115 clinically anxious adolescents aged 12 to 18 years, and their parent(s). Adolescents were randomly assigned to NET, CLIN or WLC conditions. The treatment groups received equivalent CBT content. Clinical diagnostic interviews and questionnaire assessments were completed 12 weeks after baseline, and at 6- and 12-month follow-ups.
\end{abstract}

Results: Assessment at 12 weeks post-baseline showed significantly greater reductions in anxiety diagnoses, and anxiety symptoms for both NET and CLIN conditions compared to the WLC. These improvements were maintained or further enhanced for both forms of therapy, with minimal differences between them, at 6- and 12-month follow-ups. Seventy-eight percent of adolescents in the NET group (completer sample) no longer met criteria for the principle anxiety diagnosis at 12-month follow-up compared to $80.6 \%$ in the CLIN group. Ratings of treatment credibility from both parents and adolescents were high for NET, and equivalent to CLIN. Satisfaction ratings by adolescents were equivalent for NET and CLIN conditions, whereas parents indicated slightly higher satisfaction ratings for the CLIN format.

Conclusions: Online delivery of CBT, with minimal clinician support is as efficacious as the same therapy content delivered on a face-to-face basis within a clinic setting in the treatment of anxiety disorders among adolescents. This approach offers a credible alternative to clinic-based therapy, with benefits of reduced therapist time, and greater accessibility for families who have difficulty accessing clinic-based CBT.

KEY WORDS: ADOLESCENT, ANXIETY, COGNITIVE BEHAVIOR THERAPY, COMPUTER, ONLINE 


\section{A randomized controlled trial of online versus clinic-based CBT for adolescent anxiety}

It has been well established that anxiety disorders in children can be treated efficaciously with cognitive-behavior therapy (CBT), with average remission rates of between $56 \%$ and $67 \%$ at post-treatment (Cartwright-Hatton, Roberts, Chitsabesan, Fothergill, \& Harrington, 2004; James, Soler, \& Weatherall, 2008; Silverman, Pina, \& Viswesvaran, 2008). An issue of concern, however, is that only around $25 \%$ of clinically anxious young people receive professional help (Essau, Conradt, \& Petermann, 2000; Keller et al., 1992). There are various reasons for this, including the failure to recognize that there is a problem, lack of knowledge about the availability of treatment, long waiting lists, lack of trained therapists, high costs of therapy, perceived stigma of attending a mental health clinic, and time constraints on the family (Stallard, Udwin, Goddard, \& Hibbert, 2007). Clearly, there is a need for alternative modes of treatment delivery that increase the chance that young people and their parents will seek help and participate in treatment.

Computer-based CBT has been proposed as one way of increasing access to therapy for mental health problems, and has now been evaluated for a number of psychological disorders, including anxiety, with very promising results (Reger \& Gahm, 2009). To date, studies have predominantly involved adults with research into the use of such technologies with children and adolescents still in its infancy. Computer-based therapies may be particularly appropriate for young people. They can be accessed at any time and offer a sense of privacy and confidentiality that is highly valued by adolescents (James, 2007; Stallard, Velleman, \& Richardson, 2010). Indeed, adolescents demonstrate a preference for text-based (email) or online counseling over talk-based (telephone) counseling (King, Bambling, Reid, \& Thomas, 2006) and a large proportion report using computers to search the web for information relating to worries, unhappiness or other problems (Stallard et al., 2010). Finally, communication applications on the internet, such as email, instant messaging, blogs and chat rooms have become a very important and familiar component of the lives of many adolescents 
(Subrahmanyam \& Lin, 2007). However, given that it is frequently parents who initiate treatment for their child, it is also important that parents find computer-based therapy to be a credible and acceptable form of treatment if it is to become widely adopted.

To date, there appear to be only three published randomized controlled trials evaluating internet-based treatments for childhood anxiety, none of which have involved adolescents (Khanna \& Kendall., 2010; March, Spence, \& Donovan, 2009; Spence, Holmes, March, \& Lipp, 2006). Spence, Holmes, March et al. (2006) compared clinic-based group CBT, the same treatment partially delivered via the internet, and a waitlist control with anxious children. The clinic and the combined clinic-internet conditions showed significantly greater reductions in anxiety symptoms from pre- to post-treatment than the waitlist, with improvements being maintained at 12-month follow-up. Subsequently, March et al. (2009) examined the efficacy of the same program when delivered fully online, with only minimal therapist contact by email and phone, in comparison to a waitlist control. Children in the internet condition showed small but significant reductions in clinician-rated anxiety severity, global assessment of functioning, and parent self-report measures of child anxiety compared to the wait-list at 12-week assessment. By 6-month follow-up, 75\% of children in the internet condition no longer met criteria for their anxiety disorder. Recently, Khanna and Kendall (2010) reported a small-scale randomized controlled trial comparing computer assisted treatment for 7-13 year old anxious children with individual, clinic-based CBT. Both treatments were associated with significantly greater reductions in anxiety than an attention placebo control condition. Improvements for both treatments continued over the 3-month follow-up period, with no difference in outcome.

Research relating to the evaluation of online or computer assisted CBT has, to date, been focused on younger children, rather than adolescents. Given the potential value of online treatment for adolescent anxiety problems, it is particularly important to determine its efficacy. The current study builds on previous research by comparing the efficacy of online delivery of CBT with clinically anxious adolescents, with clinic-based treatment and a waitlist control 
group. It also examines both youth and parental perceptions of credibility and satisfaction with the online therapy approach. Given that both parents and the young person are typically involved in the decision to seek and participate in treatment, it is important that both parties regard online CBT as a credible form of therapy.

It was hypothesized that both clinic- and internet-delivery of CBT at the 12-week posttherapy assessment point would be associated with greater reductions in anxiety than the waitlist, and post-treatment improvements would be maintained or enhanced at follow-up. It was further hypothesized that internet-delivered CBT would be perceived as a credible form of therapy by parents and adolescents, with a high level of treatment satisfaction reported by both.

\section{Method}

\section{Participants}

Participants were 115 adolescents (47 male, 68 female) with clinical levels of anxiety, aged from 12 to 18 years $(M=13.98, S D=1.63)$, and at least one of their parents. The majority of adolescents were born in Australia (91\%), with the remainder born in Europe, New Zealand, Asia, South Africa and the United States of America. Seventy-eight percent of adolescents lived with both biological parents, $14 \%$ lived with their mother, $7 \%$ with their mother and step-father, and 1\% lived with their father. The sample was relatively high in socioeconomic status, with $47 \%$ of households having an annual income greater than AUD $\$ 100,000$. In general, parents were relatively well educated with $46 \%$ of fathers and $51 \%$ of mothers having completed a university education.

In terms of a primary diagnosis, $48 \%$ of adolescents had generalized anxiety disorder (GAD), 35\% had social phobia (SoP), 13\% had separation anxiety disorder (SAD) and 4\% had a specific phobia (SP). Overall, 84\% of the sample also had a co-morbid anxiety disorder at a level of clinical significance. The mean number of anxiety diagnoses for adolescents prior to beginning treatment was $2.67(S D=1.31)$. A proportion of adolescents also met criteria for a co-morbid disorder other than anxiety (overall 18\%), including depression (2.6\%), dysthymic 
disorder (6\%), attention deficit hyperactivity disorder (9.6\%), and oppositional defiant disorder $(1.7 \%)$.

To be included in the study, adolescents were required to have a primary diagnosis of SAD, SoP, GAD or SP, be aged between 12 and 18 years, have access to a computer and the internet at home, and be able to read and write English at an age-appropriate level. While adolescents with a primary diagnosis of panic disorder (PD), obsessive compulsive disorder (OCD) or post-traumatic stress disorder (PTSD) were not eligible for inclusion in the study, these disorders were permissible as secondary diagnoses if the level of severity was assessed as insufficient to interfere with treatment integrity for the primary disorder. We did not accept these disorders as primary presenting problems as the intervention did not include elements that are normally included in the treatment of these particular problems, such as response prevention (for OCD), controlled breathing (for PD), or imaginal exposure (for PTSD).

For ethical reasons, adolescents with a mood disturbance rated 'moderately disturbing' or greater (severity rating of 6 or higher on the Anxiety Disorders Interview Schedule-Child Version; Silverman \& Albano, 1996) were excluded from the study and referred elsewhere for treatment for their mood disorder. Those with a pervasive developmental disorder, learning disorder, significant behavioral, substance abuse, suicidal ideation or current self-harm, were excluded from the study.

Of the 288 families who approached the study, 30 did not meet broad inclusion criteria at the telephone screen stage (e.g. outside age range, non-anxiety related difficulties, location, lack of internet access) and 258 were invited to provide informed consent and participate in the more detailed assessment to determine eligibility. At this point families were provided with more detailed information about the study, the nature of the random allocation and the need for willingness and capacity to participate in any of the conditions if allocated. Sixty-six families declined or failed to complete the detailed online questionnaires and diagnostic baseline assessment. Detailed information about reasons for non-participation is not available as 
families had not yet provided informed consent to participate in the study or completed baseline assessments. However, according to the interviewers’ reports, reasons typically included perceived lack time for therapy participation over the required period, transport difficulties if allocated to the clinic condition, unwillingness to agree to random allocation due to a preference for either internet or clinic therapy, or unwillingness to be on a wait-list, lack of interest in a CBT approach, and preference for medication treatment from a GP or psychiatrist. This left 192 families who were willing and able to participate in the study. Following baseline assessment, a further 72 were then excluded on the basis of diagnostic exclusion criteria described above and 5 families declined further participation for personal reasons. The remaining 115 families met all inclusion criteria, provided informed consent, and were randomly allocated to an experimental condition. The flow through and retention of participants at each phase of the study are summarized in Figure 1.

\section{Measures}

For ease of interpretation, measures used in this study were grouped into primary and secondary outcome measures. These measures are described below.

\section{Primary outcome measures (Diagnostic status and severity).}

\section{Anxiety Disorders Interview Schedule for DSM-IV: Child and Parent versions.}

Diagnostic status was assessed using the Anxiety Disorders Interview Schedule - Child and Parent versions (ADIS-C/P:Silverman \& Albano, 1996), administered over the telephone. Administration of the ADIS-C/P over the telephone has been demonstrated to have comparable validity to in-person administration and also has high inter-rater reliability (Cobham, Dadds, \& Spence, 1999; Lyneham \& Rapee, 2005). Assessors independent of the study and blind to treatment condition conducted the assessments. Assessors were psychologists with a minimum of 8 hours training in the use of the interview schedule and who received ongoing weekly supervision. A combined report was formed by the interviewer to reflect the responses provided by both the adolescent and the parent. Each diagnosis was assigned a clinician 
severity rating (CSR) from 0 to 8 ( 0 = absent, 8 = very severely disturbing or disabling), with scores of 4 and above representative of clinical levels of impairment. When instances occurred in which there were two or more diagnoses with the same severity rating, decisions regarding which diagnosis would be taken as primary were determined by the interviewing clinician based on a fine-grained analysis of the degree of impairment and severity of the presenting symptoms for each disorder, based on parent- and child-report on the ADIS. Prior to treatment, the mean clinical severity rating of the primary presenting anxiety disorder for the overall sample was $6.00(\mathrm{SD}=0.87)$, indicative of 'markedly disturbing/disabling' levels of anxiety.

A random sample of $15 \%$ of adolescent and parent interviews was used to determine diagnostic reliability. There was high inter-assessor agreement, with a kappa of 0.94 for the primary diagnosis and a correlation of .92 for the severity ratings between the initial interviewer and second, independent assessor.

The Children's Global Assessment Scale. The blind assessor also completed the Children’s Global Assessment Scale (CGAS: Schaffer et al., 1983). Scores ranging between 81 and 100 on the CGAS represent normal levels of functioning, scores of 61-80 indicate slight disability, moderate disability is represented by scores between 41 and 60, and scores of 1-40 indicate serious disability (Schaffer et al., 1983). The CGAS has demonstrated good inter-rater reliability $(r=.84)$ and test-retest reliability $(r=.85)$ (Dyrborg et al., 2000; Rey, Starling, Wever, Dossetor, \& Plapp, 1995; Schaffer et al., 1983). Inter-assessor reliability for the CGAS in the present study was .91 between the initial interviewer and the independent assessor.

\section{Secondary outcome measures (Child- and parent-reported anxiety}

questionnaires). The Spence Children’s Anxiety Scale (SCAS: Spence, 1998, 1999) child (SCAS-C) and parent (SCAS-P) versions were employed to measure level of adolescent anxiety. The SCAS scales include 38 items reflecting symptoms of anxiety scored for frequency of occurrence: 0 (never) to 3 (always), and include six subscales measuring panic/agoraphobia, social phobia, separation anxiety, generalized anxiety, obsessions/ 
compulsions and fear of physical injury. The SCAS-C and SCAS-P have sound psychometric properties, with internal consistency reported at .89 for the total parent anxiety score and .92 for the total child score (Muris, Schmidt, \& Merckelbach, 2000; Nauta et al., 2004; Spence, 1998; Spence, Barrett, \& Turner, 2003).

Adolescent internalizing symptoms were measured using the internalizing subscales of the Child Behavior Checklist (CBCL: Achenbach \& Rescorla, 2001) and the Youth SelfReport (YSR: Achenbach \& Rescorla, 2001). Responses are rated on a three-point scale (0 = never, 1 = sometimes, 2 = often) with higher scores indicating greater levels of symptoms. The psychometric properties of both questionnaires have been well established (Achenbach \& Rescorla, 2001).

Treatment expectancy and credibility ratings. Treatment expectancy and credibility ratings were collected from adolescents and parents following completion of the first treatment session. The measure used in the current study was adapted from a measure originally developed by Spence, Holmes, March, et al., (2006) and amended to suit the online intervention. Consisting of five items relating to expectations about the intervention (e.g. How much do you expect this program will help you beat your fears and worries?), the scale asked participants to rate their agreement with each item on a 10 -point scale $(0=$ not at all, $9=$ completely). Items were summed to form a total score, with higher scores representing greater treatment outcome expectancy and perceived credibility of the program.

Treatment satisfaction. At the 12-week assessment point, adolescents and parents were asked to rate their satisfaction with the intervention. The satisfaction scale has been previously described by Spence and colleagues (Spence, Holmes, March, et al., 2006), who adapted the questionnaire from an 11-point scale originally developed by Cobham et al. (1999). The questionnaire consisted of eight items measured on a five-point scale $(1=$ not at all, $5=$ very much). Scores were averaged to provide a mean satisfaction score. Higher scores were indicative of a higher level of satisfaction with the intervention. 


\section{Procedure}

Data collection occurred across three sites from 2005-2008: The University of Queensland and Griffith University in Brisbane, Australia, and Macquarie University in Sydney, Australia. Recruitment, assessment, intervention and follow-up procedures were identical across the three sites. Participants were recruited through advertisements in school newsletters, newspaper articles, television and radio interviews, and through referral from school guidance officers, general practitioners and other mental health professionals. An initial screening interview was conducted over the telephone with parents of potential participants. Adolescents who met broad inclusion criteria, together with at least one of their parents, were then provided with information about the study and asked to provide online, informed consent, to complete a battery of online questionnaires, and to participate in diagnostic interviews. The ADIS-C/P was then conducted via the telephone with adolescents and parents to determine the primary diagnosis and level of anxiety severity and to confirm eligibility criteria.

Families who met all inclusion criteria following diagnostic assessment and who provided informed consent were randomly assigned to one of three treatment conditions; clinic-based treatment (CLIN), internet-based treatment (NET) or a waitlist control condition (WLC). Order of random assignment was established ahead of time through a computer program and implemented by a research team member not involved in the recruitment process. Fewer young people were assigned to the WLC condition as a large effect size between the WLC and treatment conditions was expected and fewer numbers were required for adequate power in statistical analyses. Recruitment was limited to Brisbane and Sydney metropolitan areas to allow for random allocation of participants to either CLINIC or NET conditions.

The online questionnaires and telephone diagnostic interviews were re-administered approximately 12 weeks after baseline by which time it was predicted that participants should have completed the 10, weekly therapy sessions. After this point, the WLC were offered the online intervention and ceased to be part of the study. Interviews and questionnaire packages 
were re-administered at 6- and 12-month follow-up for NET and CLIN conditions. This study was conducted in accordance with the University of Queensland, Macquarie University and Griffith University Human Ethics Committees.

\section{Content of the Interventions}

Internet-based treatment (NET). Participants in the internet-based treatment condition completed BRAVE for Teenagers - ONLINE (Spence, Holmes, \& Donovan, 2006). A detailed description of the BRAVE interventions has been provided elsewhere (March et al., 2009; Spence et al., 2008), and therefore the following contains only a brief overview. The intervention is based on theoretical and empirical research relating to the psychosocial determinants of child anxiety (Dadds \& Roth, 2001; Rapee \& Spence, 2004; Silverman \& Treffers, 2001) and evidence-based, cognitive-behavioral interventions (Barrett, 1998; Rapee, Wignall, Hudson, \& Schniering, 2000; Spence, Donovan, \& Brechman-Toussaint, 2000).

The content, length and number of session activities in the internet program are designed to replicate those of the clinic-based version of the program. It targets four types of anxiety: social anxiety, generalized anxiety, separation anxiety and specific phobias. Sessions incorporate standard CBT anxiety management strategies including: psychoeducation, relaxation training, recognition of the physiological symptoms of anxiety, cognitive strategies of coping self-talk and cognitive restructuring, graded exposure, problem solving, and selfreinforcement. Parent sessions focus on the acquisition of the above mentioned CBT techniques, in addition to parenting strategies to empower parents to help their adolescents to implement anxiety management skills and to effectively deal with situations in which their adolescent becomes anxious.

Sessions include explanations of CBT techniques, real-life examples to demonstrate how each skill can be implemented and an opportunity for the participant to apply the technique to their own anxiety-provoking situations. Homework exercises are set following each session and are reviewed at the beginning of the next session. The sessions are designed 
to be engaging, interactive, and age-appropriate. Eye-catching graphics, sounds, games and quizzes are used to maintain the adolescents’ level of interest. Information is presented through interactive exercises in which material is presented followed by quizzes and tasks that check for correct understanding and provide corrective feedback where required. To avoid activities being skipped, most exercises require a response to be given in order for the adolescent or parent to move on to the next page of the session. The content of the intervention is designed to meet the developmental and cognitive level of teenagers, with age-appropriate scenarios, examples, and activities (example situations include school exams, job interviews, dating, and oral presentations). Throughout the program peer modeling is utilized to teach therapeutic skills. Adolescent characters introduced in the first treatment session are used throughout the program to depict the target anxiety disorders and demonstrate implementation of therapeutic skills in a variety of settings. The program also uses age-appropriate terminology to maximize client engagement (e.g. cognitive restructuring is referred to as 'reality checking'). Furthermore, more advanced cognitive concepts were introduced in the adolescent program, such as the role of cognitive distortions. A full discussion of developmental considerations addressed in the construction of the program is provided by Spence and colleagues (2008). The program is delivered through 10 weekly sessions for adolescents and 5 sessions for parents, each of approximately 60 minutes in duration. At one month and three months following treatment, booster sessions are provided for parents and adolescents to consolidate previously acquired skills. Sessions are accessed via a password protected website and are completed sequentially (and intended to be at intervals of 7 days). The structured nature of CBT makes it relatively straightforward to adapt session and homework content from a clinic modality to internet delivery. The greatest challenge in transferring the program from clinic to internet delivery is in the development of the exposure hierarchy. This session provides interactive information to assist the young person to design their hierarchy, with illustrations of hierarchies relevant to different types of anxiety using the 
hypothetical anxious teenager characters mentioned above, and quizzes that check for correct understanding. Instructions are given for completion of exposure tasks between sessions (in vivo) with exposure task implementation and anxiety levels before, during and after exposures being recorded within a diary kept by the young person. The outcomes of exposure are then submitted in the subsequent session in response to set computer-based questioning. Adolescents also receive a 15 minute phone call following session 5, to provide advice and assistance in regards to the construction and implementation of exposure hierarchies.

Prior to treatment, each family is assigned a therapist (BRAVE Trainer) who monitors their progress through the program and provides brief email feedback following each session. Client responses to all session and homework activities are stored in an administrator section of the program that is accessible to the therapist. Based on this information, the therapist constructs a brief feedback email to the client after each session. In addition, personalised, automated computer-generated emails are sent on behalf of the online therapist to congratulate participants for completion of sessions and to provide feedback about responses to quiz tasks. Seven days after completion of each session, personalised automated reminder emails are sent, advising that the next session is available for completion. Finally, personalized, automated reminder emails are sent if the session is not accessed within two days of becoming available.

Clinicians were provided with set criteria and standard templates to use when responding by email to each session and could also insert personalized information relating to session tasks. All therapists were psychologists who had received a minimum of two days training with the BRAVE-ONLINE materials. In addition, therapists were provided with weekly supervision from an experienced clinical psychologist. During supervision, the therapist's online responses were reviewed in order to maintain a high standard of integrity and to ensure that each therapist was adhering to all guidelines for participant contact (e.g., length and content of session responses, adhering to templates). 
Clinic-based treatment (CLIN). In the clinic condition, participants received the equivalent program to the online therapy in terms of content, length and number of session activities, but all sessions were conducted face-to-face with a therapist within a clinic setting. BRAVE-CLINIC is a manualized individual CBT program for adolescent anxiety (Donovan, Holmes, \& Spence, 2006) adapted from the child program evaluated by Spence, Holmes, March et al. (2006). Adolescents and parents received a workbook to use during the sessions and a copy of the relaxation CD.

Clinic sessions were conducted by registered Psychologists at the University of Queensland, Griffith University and Macquarie University Psychology Clinics. All clinicians received two days of training in the manualized clinic program. Training was conducted by the program coordinators who were also responsible for providing ongoing weekly supervision for all clinicians. Following each session, clinicians were required to complete a checklist of activities completed with the client. Further, a random subset of $15 \%$ of clinic sessions were video recorded for each client, and were watched by an independent observer/therapist to confirm fidelity of implementation. On average, 95.61\% of activities that should have been completed were completed each session.

Wait-list control condition (WLC). Waitlist participants were assessed at baseline and then again after a 12-week waiting period. The WLC did not have any form of planned contact with the project team during the 12-week period. They were provided with contact details for the researchers in the event that serious clinical deterioration in symptoms took place, although none of the participants made such contact. Following this period, WLC participants were offered NET treatment and subsequently ceased to be part of the study.

\section{Results}

\section{Power}

Power calculations were based on chi-square tests using diagnostic status as the primary outcome measure. The study was powered on the comparison between the two active 
treatment conditions as this comparison required the most power. It was calculated that a sample size of 44 participants per active treatment group would provide a power of .80 to detect a clinically meaningful (medium) effect size of .30, at an alpha of .05.

\section{Statistical Analysis}

Primary outcomes for the investigation were clinical diagnoses (based on the ADIS$\mathrm{C} / \mathrm{P}$ ), clinician severity ratings (CSR), and clinician-rated global functioning (CGAS). Secondary outcomes included parent ratings of anxiety symptoms on the SCAS-P and internalizing problems on the CBCL, plus youth self-report on the SCAS-C and the YSR.

Analyses for the continuous variables were conducted using an intention-to-treat (ITT) approach, and included all participants who were randomly assigned to conditions. Mixed model, repeated measures analyses using SPSS Mixed were used separately to evaluate the continuously scaled outcome measures over time as a function of treatment condition. Maximum likelihood estimation was used to determine parameter estimates. Treatment condition was regarded as a fixed between groups effect, with time taken as a fixed, repeated measure, within-subjects effect, and the intercept as a random effect. Main effects for treatment condition were based on estimated scores at each time point. For categorical diagnostic results, data were examined for ITT and completer samples, using Chi-Square tests.

The analyses first examined differences between the three experimental conditions (WLC, NET and CLIN) from baseline to the 12-week assessment point. Then, because the WLC participants were not included in the follow-up component of the study, separate analyses were conducted across 4 assessment occasions (baseline, 12-week assessment, and 6and 12-month follow-ups) comparing the NET and CLIN conditions.

\section{Baseline Comparisons}

Initial differences between the three experimental conditions were examined for baseline demographic and treatment outcome measures. No significant differences were evident for demographic variables including age, gender, family income, or parental education. 
Significant baseline differences were evident across conditions however for the primary outcome measures of clinician severity rating $\left(\mathrm{CSR} ; F(2,112)=5.12, p=.007, \eta^{2}=.084\right)$, and CGAS $\left(F(2,112)=4.41, p=.014, \eta^{2}=.072\right)$. Post-hoc contrasts (Scheffe) indicated significantly higher anxiety severity on the CSR $(t=-3.07, d f=112, p=0.01)$ and poorer functioning on the CGAS $(t=2.96, d f=112, p=0.02)$ in the CLIN condition compared to the WLC condition at baseline but not between CLIN versus NET, or NET versus WLC conditions.

\section{Impact of Missing Data}

There were no significant differences in baseline levels of primary and secondary outcome variables or demographic characteristics of the child and family between participants who were retained in the study compared to those who were missing at one or more assessment occasions. Dummy variables were computed for the primary outcome measures in which the number of occasions in which there was a missing value on that variable was computed. There were no significant interactions between missingness and the condition by time interaction, nor with time or condition for either CSR or CGAS ratings.

\section{Treatment Expectancy and Credibility Ratings}

No significant differences in adolescent outcome expectancy and perception of treatment credibility at the end of the first treatment session were found between the two treatment groups, $t(81)=-0.91, p=.37$, two-tailed $($ NET $M$ item score $=6.57, S D=1.49$; CLIN $M$ item score $=6.88, S D=1.60$ ), where scores ranged from 0-9. Similarly, there was no significant difference in treatment expectation and perception of treatment credibility between parents in NET and CLIN conditions, $t(83)=-1.11, p=.27$, two-tailed, (NET $M$ item score = 7.08, $S D=1.05 ;$; CLIN $M$ item score $=7.30, S D=0.75$ ) These ratings are similar to those found for a previous internet-based intervention using the same measure (March et al., 2009) and indicate a moderate to strong expectancy for positive outcome, and credibility of both treatment approaches. 


\section{Completion of Therapy Sessions}

The average number of sessions completed by NET participants at the 12-week assessment point was 7.5 out of 10 for adolescents and 4.48 out of 5 for parents, although only $39 \%$ of adolescents and $66 \%$ of parents had completed all of their treatment sessions at this point. This was not significantly different to that of CLIN families, who completed on average, 8.25 out of 10 sessions for adolescents and 4.43 out of 5 sessions for parents, with $57 \%$ of adolescents and 70\% of parents completing all treatment sessions by the 12 -week assessment. Families in both conditions completed further sessions over the next few weeks. The rate of session completion was, however, significantly slower in the NET than the CLIN group, with a mean rate of session completion of one session per $14.00(S D=7.28)$ days for the NET participants compared to 1 per $8.96(S D=3.09)$ days for the 32 CLIN adolescents from whom these data were available, $\mathrm{F}(1,74)=13.50, \mathrm{p}<.001$, eta $\mathrm{sq}=.15$.

By 12-month follow-up, adolescents in the NET condition had completed an average of 8.20 out of 10 sessions and parents had completed 4.70 out of 5 sessions, with $57 \%$ of adolescents and $79 \%$ of parents completing all treatment sessions. In the CLIN condition, by 12-month follow-up, adolescents had completed an average of 8.70 out of 10 sessions and parents had completed 4.50 out of 5 sessions, with $79 \%$ of adolescents and $79 \%$ of parents completing all sessions. No significant differences were evident between NET and CLIN adolescents or parents in terms of the mean number of sessions completed by 12-month follow-up. However, significantly fewer NET adolescents had completed all 10 sessions by 12-month follow-up, compared to the CLIN group, $\chi^{2}(1, N=88)=5.24, p=.02$.

\section{Outcome: Baseline to 12-week Assessment Effects}

Primary outcome measures - Diagnostic data. Diagnostic status was determined on the basis of combined parent and adolescent reports on the ADIS-P and -C. Diagnostic status was considered both in terms of the percentage of children who no longer met DSM-IV criteria for their primary anxiety disorder and for any anxiety disorder. For this categorical data, 
results are reported for both completer and ITT samples (see Table 1). For the purposes of the ITT sample, participants without 12-week assessment data were assumed to still possess their pretreatment diagnoses at 12-weeks. For the completer sample, analyses were conducted with participants for whom diagnostic data was actually available at the 12-week assessment.

Primary anxiety disorder - clinically significant improvement. Clinically significant improvement was examined using the percent of adolescents who were free of their primary diagnosis at 12-week assessment. The criterion for diagnosis free required the CSR for the primary diagnosis to be at a subclinical level (3 or lower). When considering the ITT sample, a significant difference was found between conditions (see Table 1$), \chi^{2}(2, N=115)=8.90, p=$ .01. Post-hoc comparisons revealed significant differences between the WLC and NET conditions, $\chi^{2}(1, N=71)=8.85, p=.003$ and between WLC and CLIN conditions, $\chi^{2}(1, N=$ $71)=7.06, p=.008$, but not between NET and CLIN groups, $\chi^{2}(1, N=88)=.21, p=.65$.

Similar results were found for the completer sample, with a significant overall difference between conditions, $\chi^{2}(2, N=105)=8.73, p=.01$. Post-hoc comparisons for the completer sample revealed significant differences between the WLC and NET conditions, $\chi^{2}$ $(1, N=65)=8.57, p=.003$ and between WLC and CLIN conditions, $\chi^{2}(1, N=64)=7.05, p$ $=.008$, but not between NET and CLIN groups, $\chi^{2}(1, N=81)=.15, p=.70$.

Any anxiety disorder. An evaluation of the percentage of adolescents in the ITT sample who no longer met criteria for any anxiety disorder at the 12-week assessment failed to find a significant difference between the three conditions, $\chi^{2}(2, N=115)=3.90, p=.14$ (see Table 1). For the completer sample, there was also no significant difference between conditions, $\chi^{2}$ $(2, N=105)=3.82, p=.15$.

Primary outcome measures - Continuous measures. A linear mixed model analysis was conducted to compare the three conditions over time from baseline to the 12-week assessment for primary and secondary continuous outcome measures. Given the significant baseline differences between conditions, analyses were initially conducted using the baseline 
CSR as a covariate. However, as there was no significant effect of baseline CSR upon change over time in the primary outcome measures, the analyses reported here do not include baseline CSR as a covariate in order to facilitate interpretation of the data. Table 2 summarizes the estimated marginal means for primary and secondary outcome measures.

For the primary outcome measures, the analyses conducted on CSR scores revealed a significant effect for treatment condition, $F(2,116.35)=3.63, p=.03$, time, $F(1,113.27)=$ 91.42, $p<.001$, and a significant treatment by time interaction, $F(2,113.14)=15.58, p<.001$. Post-hoc contrasts revealed significant changes over time for the $\operatorname{NET} F(1,111.85)=72.87$, $p<.001$ and CLIN conditions $F(1,113.09)=83.39, p<.001$, but not for the WLC. Results pertaining to fixed effects for intercept and slopes and effect sizes are shown in Table 3, and indicate that both NET and CLIN conditions decreased in CSR scores significantly more than the WLC from baseline to 12-week assessment.

For the CGAS ratings, there was no significant effect for treatment condition, $F$ $(2,117.12)=1.11, p=.33$, but a significant effect for time, $F(1,111.36)=124.52, p<.001$, and a significant treatment by time interaction, $F(2,111.29)=18.49, p<.001$. Post-hoc comparisons indicated no significant change for the WLC, but significant increases (improved functioning) in CGAS scores for NET $F(1,110.34)=105.51, p<.001$ and CLIN groups $F$ $(1,111.25)=99.34, p<.001$. Both the NET and CLIN conditions showed significantly greater increases than the WLC in CGAS scores from baseline to 12-week assessment (see Table 3).

Secondary outcome measures - Continuous measures. For the secondary outcome measures, significant effects were found for time on the SCAS-C, $F(1,102.06)=63.43, p<$ .001 , with CLIN $F(1,100.76)=33.58, p<.001$, NET $F(1,102.73)=24.90, p<.001$ and WLC $F(1,102.41)=12.04, p<.01$, all showing a significant reduction in symptoms over time. The parent report SCAS-P also showed a significant time effect, $F(1,103.19)=10.97, p<.001$, although post-hoc analyses revealed that only the CLIN condition reported a significant reduction in symptoms over time $F(1,104.62)=3.27, p<.001$. For the CBCL and YSR, there 
were significant effects for time, $F(1,109.80)=38.46, p<.001$, and, $F(1,101.78)=75.90, p<$ .001, respectively. Post-hoc contrasts indicated that those in the CLIN condition showed a significant reduction in scores over time on the CBLC, $F(1,111.07)=29.05, p<.001$, and YSR, $F(1,101.70)=36.12, p<.001$. The NET condition also showed significant reductions in scores on the CBCL over time, $F(1,107.13)=23.10, p<.001$, and the YSR, $F(1,99.69)=$ 54.18, $p<.001$. In contrast, the WLC showed no significant reduction over time for the CBCL, but a small yet significant reduction in scores was evident for the YSR, $F(1,103.02)=6.97, p$ $<.05$, from baseline to 12 -week assessment. There were no significant condition by time interactions or condition effects from baseline to 12-week assessment on any of the secondary outcome measures. When adolescent gender was included in the model, there were no significant interaction effects between gender, condition and time.

\section{Outcome: Baseline to 12-week Assessment and 6- and 12-month Follow-ups}

Primary outcome measures - Diagnostic data. Follow-up data were examined only for the NET and CLIN treatment conditions, as the WLC participants were not available beyond the 12-week assessment point. For the purposes of the ITT sample, missing data at 6and 12-month follow-up were replaced with the value from the previous time point at which they provided assessment data.

Clinically significant change at 6-month follow-up. The percentage of adolescents in the ITT sample who no longer met DSM-IV criteria for their primary anxiety disorder continued to increase from 12-week assessment to 6-month follow-up, for both NET and CLIN conditions (see Table 1), suggesting that diagnostic status was further improved over time. Similar improvements were evident for both treatment conditions in the completer sample. There were no significant differences between treatments for either the ITT, $\chi^{2}(1, N=88)=$ $0.18, p=.67$, or completer samples, $\chi^{2}(1, N=73)=0.11, p=.74$. Participants also continued to improve at 6-month follow-up with respect to the presence of any anxiety disorder (see 
Table 1). Again, there were no significant differences between treatment conditions for either the ITT, $\chi^{2}(1, N=88)=0.19, p=.67$ or completer samples, $\chi^{2}(1, N=73)=0.12, p=.73$.

Clinically significant change at 12-month follow-up. Diagnostic improvements continued to be seen from 6- to 12-month follow-up for both treatment conditions in the ITT and completer samples (see Table 1), with no significant treatment differences for either the $\operatorname{ITT}, \chi^{2}(1, N=88)=0.00, p=1.00$ or completer samples, $\chi^{2}(1, N=73)=0.05, p=.82$ in terms of primary anxiety disorder. This pattern of continued improvement was also found for any anxiety disorder (see Table 1), again with no significant difference between conditions, for either the completer or ITT samples, $\chi^{2}(1, N=88)=0.19, p=.67$ and $\chi^{2}(1, N=73)=0.84, p$ $=.36$, respectively.

Primary outcome measures - Continuous measures. A linear mixed model analysis was then used to examine differences in primary outcome measures across the four time points for the two treatment conditions. Outcomes at each assessment occasion are reported in Table 4. There was a significant effect for time for CSR ratings, $F(3,238.17)=139.05, p<.001$, and the CGAS, $F(3,236.01)=173.81, p<.001$. There were no significant effects for condition or between condition and time for the primary outcome measures. Table 5 provides the parameter estimates and effect sizes for the mixed model analysis. Thus, both CLIN and NET conditions decreased in anxiety severity and increased in overall functioning to the same extent over time. When gender of child was included in the models, there were no significant interaction effects between gender, condition and time.

Secondary outcome measures - Continuous measures. There was a significant main effect of time on the SCAS-C, $F(3,217.27)=96.11, p<.001$, the SCAS-P, $F(3,217.08)=$ 61.27, $p<.001$, the CBCL - Internalizing, $F(3,216.69)=55.91, p<.001$, and the YSR Internalising, $F(3,219.12)=66.31, p<.001$ scores. However, there were no significant condition, or condition by time interaction effects, indicating that adolescents in both conditions showed significant and equivalent decreases in anxiety symptoms over time. Table 
5 shows the parameter estimates and effect sizes for the mixed model analysis and demonstrates that there were no significant differences in changes over time between the CLIN and NET conditions for the secondary outcome measures.

\section{Satisfaction with the Program}

Satisfaction data was collected for 40 adolescents and 41 parents in the NET condition and for 36 adolescents and 37 parents in the CLIN condition. For adolescent reports of treatment satisfaction, there were no significant differences between the NET $(M=3.53, S D=$ 0.73) and CLIN $(M=3.79, S D=0.95)$ conditions, $t(76)=-1.36, p=.18$, two-tailed. However, parents in the CLIN condition $(M=3.99, S D=0.69)$ reported significantly higher satisfaction with the program than parents in the NET condition $(M=3.57, S D=0.81), t(78)=-2.42, p=$ .02, two-tailed. Overall, the results indicate that adolescents and parents reported moderate to high satisfaction with the treatment received, although parents in the CLIN condition reported slightly higher program satisfaction.

\section{Discussion}

The results of the study demonstrate the significant benefits of online delivery of CBT for the treatment of anxiety disorders amongst adolescents, with outcomes at 12 month followup being similar to those found for clinic-based therapy. Furthermore, the online program was regarded as a highly credible approach by both parents and adolescents, at a level equivalent to clinic-based CBT, and produced moderate to high ratings of satisfaction with treatment. Both internet and clinic-based CBT produced significantly greater reductions in clinician-rated anxiety and greater improvements in overall functioning than the waitlist group at the 12-week assessment point, with no significant difference between treatment formats. The short-term effects were equivocal for the self-report questionnaires completed by the parents and adolescents.

Overall, the outcomes for both the CLIN and NET groups at 12-week post-baseline tended to be somewhat lower than those reported for clinic-based therapy (Cartwright-Hatton, 
et al., 2004; James, et al., 2008; Silverman, et al., 2008). The rates are also lower than the posttreatment remission rates reported by the same research team using the same intervention with a younger sample using a combined clinic-internet conditions (Spence, Holmes, March, et al., 2006). They are, however, consistent with those reported in a recent study by Hudson et al. (2009) in which 45\% of the 7-16 year old sample were free of their primary anxiety diagnosis immediately after CBT. Similarly, the extent of the clinically significant improvements in the CGAS ratings for the NET and CLIN conditions at 12-weeks are consistent with those reported by Walkup et al. (2008) over the same time period for clinic-based CBT with 7 - 17 year olds. It may be that anxiety disorders are of longer duration in the older age group and possibly more difficult to treat. Alternatively, the weak results at 12-weeks may reflect the finding that a significant number of families in both the clinic and internet condition had yet to complete all 10 sessions by this point. This finding is consistent with that reported by March et al. (2009) using online therapy with children, but is somewhat unexpected for the CLIN condition. Post-assessments were conducted at 12-weeks post baseline assessment regardless of whether participants had completed all sessions to allow comparisons with results at the same time point across the three conditions, and with other clinic based efficacy trials in this area. It is difficult to determine whether the rates of adolescent therapy session completion in the present study are different to those in the clinic-based literature, given that such information is rarely reported. It may be that adolescents are more reluctant, busier or less motivated than younger children and that this contributes to the lower than expected rates of session completion and, in turn, with lower rates of improvement. The teenagers in both treatments did, however, continue to complete sessions over the next few weeks, although those in the NET condition were less likely to have completed all 10 sessions by 12 -month follow-up.

The results indicate that over the 12-month follow-up period, the majority of young people continued to improve in both treatment conditions, with no significant differences in 
treatment outcome across therapy formats. It appears therefore that even though the internet participants were less likely to have completed all 10 sessions, their clinical outcome was equivalent to that found for the clinic-based group in the longer term. By 12-month follow-up, $78.4 \%$ and $80.6 \%$ of young people in the NET and CLIN conditions respectively in the completer sample were free of their primary diagnosis, indicating a level of improvement that is consistent with the results for CBT at a similar follow-up point elsewhere in the literature (Cartwright-Hatton, et al., 2004; James, et al., 2008; Silverman, et al., 2008). Although the rates relating to the ITT sample were lower, again these were consistent with results reported in other studies for clinic-based therapy. In addition to improvements in diagnostic status, both conditions showed continued and significant improvements in anxiety symptoms according to both parent and youth reports, and significant enhancements in overall functioning on the clinician-rated CGAS.

It should be noted however, that even though both conditions showed equivalent and strong effects upon the primary diagnoses, the effects were weaker in terms of change in "any anxiety diagnosis” with $62.2 \%$ of NET and $72.2 \%$ of CLIN completers no longer experiencing any anxiety diagnoses. The difference between conditions was not statistically significant, but it is of concern that a significant proportion of participants in both conditions still experienced at least one clinically significant anxiety problem at 12 -month follow up (37.8\% in the NET and $27.8 \%$ in the CLIN conditions). This point tends to be masked if reliance is placed on the CSR of the primary diagnosis as the main outcome measure. For this reason, future studies should consider using an indicator of improvement which takes into account functioning across anxiety problems in general, such as the Clinician Global Impressions - Improvement rating (Guy \& Bonato, 1970). It is feasible that both clinic and internet CBT of the length and type of content used in this study may not be sufficient to bring about significant improvements across the broad spectrum of anxiety symptoms in those young people with more complex, comorbid presentations. Indeed, Walkup et al., (2008) found that a combination of CBT and medication 
(sertraline) was more effective than either approach alone using the CGI-I as the main outcome measure, suggesting that CBT alone may not be sufficient for some clients.

One of the encouraging findings in the present study was that the internet program was evaluated positively by the participants. Adolescent consumer ratings indicated a high degree of satisfaction with the therapy, and there were no significant differences between clinic and internet delivery groups in the degree of program satisfaction. However, although the difference was small and all parents rated the online program positively, parents in the NET condition rated the treatments significantly lower than did parents in the CLIN format,. It appears that parents do still prefer face-to-face contact with a therapist when their child is being treated for anxiety.

The study had several strengths that should be noted. It appears to be the first randomized controlled trial comparing internet versus clinic delivery of CBT for anxiety disorders that focuses specifically upon an adolescent sample and that includes a waitlist control. The methodology included blind assessment of diagnostic status, strong fidelity checks for quality of implementation, and a sample size that provided sufficient power to enable a valid comparison between treatments.

There are, however, some limitations in the design of the study. The first relates to the timing of assessments and equivalence of therapy completion across conditions. Although the use of common assessment time points across conditions can be regarded as a strength of the study, it must be noted that a significant proportion of adolescents in both the NET and CLIN conditions had not completed all 10 therapy sessions by the 12-week assessment. Although participants in both conditions continued to complete sessions over the following weeks, NET participants tended to work more slowly through their sessions and were less likely to have completed all 10 sessions by the final assessment time point. The potential implications of these confounds must be considered. On the one hand, it is possible that the results for the NET condition may have been stronger had more of the NET participants completed all 10 sessions. 
On the other, it is feasible that the clinic-based CBT results may have been stronger if the spacing between sessions had been longer in the CLIN condition, facilitating consolidation of coping skills. These issues present some interesting themes for future research, such as identifying ways of increasing the proportion of online participants who completed all sessions, and examining whether clinic-based CBT may be more effective if the time between therapy sessions is extended. It will also be important to determine the extent to which outcome from internet delivered CBT is influenced by therapy adherence (such as the number of exposures completed, number of therapy tasks completed, or time spent completing the sessions).

A further limitation of the study was the failure to include an attention placebo control group to enable conclusions to be drawn about the specific effects of treatment, over and above non-specific factors. A recent study by Hudson et al. (2009) suggests however, that effects following a non-specific intervention are significantly less than those associated with CBT with clinically anxious children and adolescents. Thus, the positive results for treatment at follow-up are unlikely to reflect non-specific factors. The design of the present study was also weakened by the absence of the waitlist condition at follow-up. It was not considered ethical to continue the waitlist condition beyond the 12-week assessment point, and participants in this condition then received the internet treatment and ceased to be part of the study. Although it cannot be said for certain that the positive outcomes at follow-up were not just reflective of spontaneous remission/natural recovery, this possibility is unlikely given that the Hudson et al. (2009) study found only $45.5 \%$ of young people in a non-specific control condition to be diagnosis free at 6-month follow-up. This value is considerably lower than that for both treatments in the present study.

Another weakness of the current study concerned the characteristics of the sample which tended to include quite well-educated parents, with relatively good incomes. Future research should examine the efficacy of such interventions with families from a broader range 
of socio-demographic backgrounds and those who are referred to community-based clinics (rather than a university research clinic), in order to determine the generalizability of the findings.

The study was also restricted to four types of primary presenting anxiety disorder, namely generalized anxiety disorder, social phobia, separation anxiety disorder or specific phobia. Those with a primary anxiety disorder involving panic disorder, obsessive-compulsive disorder or post-traumatic stress disorder were excluded. Future studies should investigate the feasibility and effectiveness of the online CBT intervention with these additional anxiety diagnoses.

In summary, the results of the present study suggest that online CBT, with minimal therapist contact, for adolescent anxiety disorders offers an efficacious alternative to clinic based treatment. Online CBT may represent one way in which a greater number of clinically anxious young people can be helped effectively than can be achieved solely through clinicbased interventions. Parents and adolescents regarded the online approach to be credible, with moderate to high levels of satisfaction. Online therapy reduces the burden of travel for families, overcomes issues of stigma associated with attending a clinic, and also requires significantly less therapist hours. In the present study, online support by the therapist took between 10-15 mins per week (for 10 therapy weeks and 2 booster sessions; total of 120 - 180 mins), compared to 60 mins per week for 10 youth and 5 parent sessions, plus 2 youth boosters and 2 parent boosters (total of 1140 mins) for clinic treatment. Potentially, a clinician could provide treatment to a larger number of families using the clinician-assisted online approach than would be possible using clinic-based therapy alone. It would be valuable in future research to conduct a more in-depth cost-benefit analysis of this approach.

\section{Acknowledgements}

The authors would like to the thank the National Health and Medical Research Council for funding this research 


\section{References}

Achenbach, T. M., \& Rescorla, L. A. (2001). Manual for the ASEBA school-age forms and profiles. Burlington, VT: University of Vermont, Research Centre for Children, Youth and Families.

Barrett, P. M. (1998). Evaluation of cognitive-behavioral group treatments for childhood anxiety disorders. Journal of Clinical Child Psychology, 27(4), 459-468.

Cartwright-Hatton, S., Roberts, C., Chitsabesan, P., Fothergill, C., \& Harrington, R. (2004). Systematic review of the efficacy of cognitive behaviour therapies for childhood and adolescent anxiety disorders. British Journal of Clinical Psychology, 43(4), 421-436.

Cobham, V. E., Dadds, M. R., \& Spence, S. H. (1999). Anxious children and their parents: What do they expect? Journal of Clinical Child Psychology, 28(2), 220-231.

Dadds, M. R., \& Roth, J. H. (2001). Family processes in the development of anxiety problems. In M. W. Vasey \& M. R. Dadds (Eds.), The developmental psychopathology of anxiety (pp. 75-81). London: Oxford University Press.

Donovan, C. L., Holmes, J., \& Spence, S. H. (2006). BRAVE for Teenagers: A program for adolescents with anxiety. School of Psychology, University of Queensland.

Dyrborg, J., Larsen, F., Nielsen, S., Byman, J., Nielsen, B., \& Gutre-Delay, F. (2000). The Children's Global Assessment Scale (CGAS) and Global Assessment of Psychological Disability (GAPD) in clinical practice - substance and reliability as judged by interclass correlations. European Child and Adolescent Psychiatry, 9, 195-201.

Essau, C. A., Conradt, J., \& Petermann, F. (2000). Frequency, comorbidity, and psychosocial impairment of anxiety disorders in German adolescents. Journal of Anxiety Disorders, 14(3), 263-279.

Guy, W., \& Bonato, R. R. (Eds.). (1970). CGI: Clinical Global Impressions. Chevy Chase (Maryland): National Institute of Mental Health.

Hudson, J. L., Rapee, R. M., Deveney, C., Schniering, C. A., Lyneham, H. J., \& Bovopoulos, N. (2009). Cognitive-behavioral treatment versus an active control for children and adolescents with anxiety disorders: A randomized trial. Journal of the American Academy of Child and Adolescent Psychiatry 48(5), 533-544.

James, A. (2007). Principles of youth participation in mental health services. Medical Journal of Australia, 187(suppl 7), s57-s60.

James, A., Soler, A., \& Weatherall, R. (2008). Cognitive behavioural therapy for anxiety disorders in children and adolescents. Cochrane Database of Systematic Reviews, Issue 4. Art. No.: CD004690. DOI: 10.1002/14651858.CD004690.pub2.

Keller, M. B., Lavori, P. W., Wunder, J., Beardslee, W. R., Schwartz C, E., \& Roth, J. (1992). Chronic course of anxiety disorders in children and adolescents. Journal of the American Academy of Child \& Adolescent Psychiatry, 31(4), 595-599.

Khanna, M. S., \& Kendall., P. C. (2010). Computer-assisted cognitive behavioral therapy for child anxiety: Results of a randomized clinical trial. Journal of Consulting and Clinical Psychology, 78(5), 737-745.

King, R., Bambling, M., Reid, W., \& Thomas, I. (2006). Telephone and online counselling for young people: A naturalistic comparison of session outcome, session impact and therapeutic alliance. Counselling \& Psychotherapy Research, 6(3), 175-181.

Lyneham, H. J., \& Rapee, R. M. (2005). Agreement Between Telephone and In-Person Delivery of a Structured Interview for Anxiety Disorders in Children. Journal of the American Academy of Child \& Adolescent Psychiatry, 44(3), 274-282.

March, S., Spence, S. H., \& Donovan, C. L. (2009). The efficacy of an internet-based cognitive-behavioral therapy intervention for child anxiety disorders. Journal of Pediatric Psychology, 34(5), 474-487. 
Muris, P., Schmidt, H., \& Merckelbach, H. (2000). Correlations among two self-report questionnaires for measuring DSM-defined anxiety disorder symptoms in children: The Screen for Child Anxiety Related Emotional Disorders and the Spence Children's Anxiety Scale. Personality \& Individual Differences, 28(2), 333-346.

Nauta, M. H., Scholing, A., Rapee, R. M., Abbott, M., Spence, S. H., \& Waters, A. (2004). A parent-report measure of children's anxiety: Psychometric properties and comparison with child-report in a clinic and normal sample. Behaviour Research \& Therapy, 42(7), 813-839.

Rapee, R. M., \& Spence, S. H. (2004). The etiology of social phobia: Empirical evidence and an initial model. Clinical Psychology Review. Special Issue: Social Phobia and Social Anxiety, 24(7), 737-767.

Rapee, R. M., Wignall, A., Hudson, J. L., \& Schniering, C. A. (2000). Treating anxious children and adolescents: An evidence-based approach. Oakland, CA: Harbinger Publications.

Reger, M. A., \& Gahm, G. A. (2009). A meta-analysis of the effects of internet- and computerbased cognitive-behavioral treatments for anxiety. Journal of Clinical Psychology, 65(1), 53-75.

Rey, J., Starling, J., Wever, C., Dossetor, D., \& Plapp, J. (1995). Inter-rater reliability of Global Assessment of Functioning in a clinical setting. Journal of Child Psychology \& Psychiatry, 36, 1228-1231.

Schaffer, D., Gould, M. S., Brasic, J., Ambrosini, P., Fisher, P., Bird, H. R., et al. (1983). A Children's Global Assessement Scale (CGAS). Archives General Psychiatry, 40, 12281231.

Silverman, W. K., \& Albano, A. M. (1996). Anxiety Disorders Interview Schedule for Children for DSM-IV: Child and Parent Versions). San Antonio, TX: The Psychological Corporation - Harcourt, Brace \& Company.

Silverman, W. K., Pina, A. A., \& Viswesvaran, C. (2008). Evidence-based psychosocial treatments for phobic and anxiety disorders in children and adolescents. Journal of Clinical Child and Adolescent Psychology, 37(1), 105-130.

Silverman, W. K., \& Treffers, P. D. A. (2001). Anxiety disorders in children and adolescents: Research, assessment and intervention. New York: Cambridge University Press.

Spence, S. H. (1998). A measure of anxiety symptoms among children. Behaviour Research and Therapy, 36, 545-566.

Spence, S. H. (1999). Spence children's anxiety scale (parent version). Brisbane: University of Queensland.

Spence, S. H., Barrett, P. M., \& Turner, C. M. (2003). Psychometric properties of the Spence children's anxiety scale with young adolescents. Journal of Anxiety Disorders, 17(6), 605-625.

Spence, S. H., Donovan, C., \& Brechman-Toussaint, M. (2000). The treatment of childhood social phobia: The effectiveness of a social skills training-based, cognitive-behavioural intervention, with and without parental involvement. Journal of Child Psychology and Psychiatry and Allied Disciplines, 41(6), 713-726.

Spence, S. H., Donovan, C. L., March, S., Gamble, A., Anderson, R., Prosser, S., et al. (2008). Online CBT in the Treatment of Child and Adolescent Anxiety Disorders: Issues in the Development of BRAVE - ONLINE and Two Case Illustrations. Behavioural and Cognitive Psychotherapy, 36(Special Issue 04), 411-430.

Spence, S. H., Holmes, J., \& Donovan, C. L. (2006). BRAVE for Teenagers - ONLINE: An internet based program for adolescents with anxiety. Brisbane, Australia: School of Psychology, University of Queensland. 
Spence, S. H., Holmes, J. M., March, S., \& Lipp, O. V. (2006). The Feasibility and Outcome of Clinic Plus Internet Delivery of Cognitive-Behavior Therapy for Childhood Anxiety. Journal of Consulting and Clinical Psychology, 74(3), 614-621.

Stallard, P., Udwin, O., Goddard, M., \& Hibbert, S. (2007). The Availability of Cognitive Behaviour Therapy Within Specialist Child and Adolescent Mental Health Services (CAMHS): A National Survey. Behavioural and Cognitive Psychotherapy, 35(04), 501-505.

Stallard, P., Velleman, S., \& Richardson, T. (2010). Computer Use and Attitudes Towards Computerised Therapy Amongst Young People and Parents Attending Child and Adolescent Mental Health Services. Child and Adolescent Mental Health, 15(2), 80-84. Subrahmanyam, K., \& Lin, G. (2007). Adolescents on the net: Internet use and well-being. Adolescence, 42(168), 659-677.

Walkup, J. T., Albano, A. M., Piacentini, J., Birmaher, B., Compton, S. N., Sherrill, J. T., et al. (2008). Cognitive Behavioral Therapy, Sertraline, or a Combination in Childhood Anxiety. The New England Journal of Medicine, 359(26), 2753-2766. 
288 families contacted the project and completed telephone screen

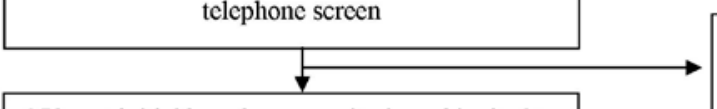

258 met initial broad screen criteria and invited to participate in detailed assessment

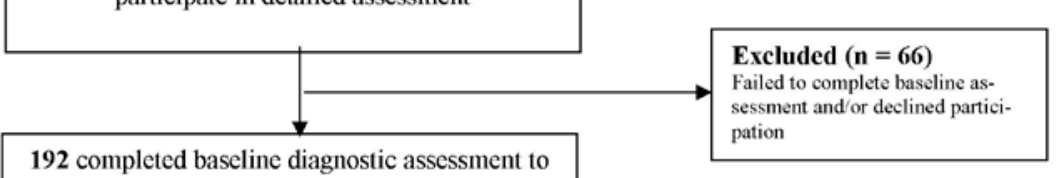

192 completed baseline diagnostic assessment to determine eligibility for inclusion

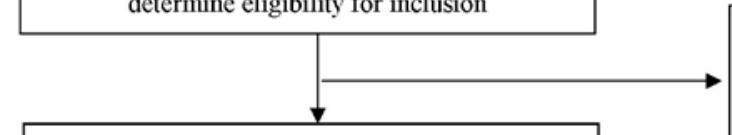

115 fulfilled inclusion criteria and randomized to: criteria

pation

Excluded $(\mathbf{n}=\mathbf{7 7})$

Subclinical anxiety $(\mathrm{n}=12)$

Other primary disorder $(n=44)$

Suspected abuse $(n-4)$

ther treatment $(\mathrm{n}-12)$

Declined further participation

following assessment $(\mathrm{n}-5)$
Excluded $(n=30)$

Did not meet broad inclusion

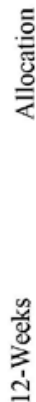

Allocated to NET condition $(n=44)$

Commenced NET condition $(n=44)$

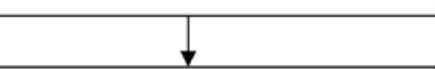

41 completed 12-week assessment

1 discontinued treatment

2 families were not available for in-

terview (completed questionnaires

and remained in study)

44 analyzed in intent-to-treat sample

37 completed
assessment

4 declined to participate further

2 families not available for interview

(but completed questionnaires)

44 analysed in intent-to-treat sample

44 analyzed in intent-to-treat sample

27 analysed in intent-to-treat sample

Allocated to WLC condition $(n=27)$

Commenced CLIN condition $(n=44)$

Commenced WLC condition $(n=27)$

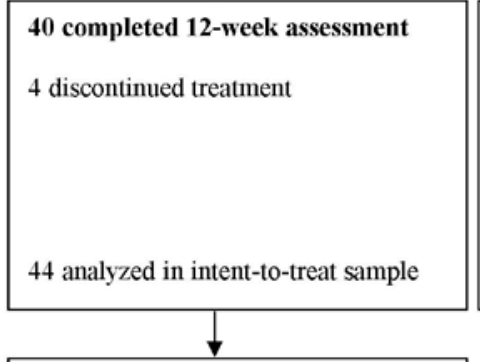

24 completed 12-week assessment

3 discontinued condition

WLC ceased participation in study and were offered treatment

36 completed 6-month follow-up assessment

4 declined to participate further

44 analysed in intent-to-treat sample

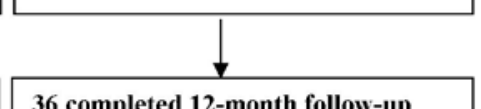

37 completed 12-month follow-up assessment

1 declined to participate further

1 family not available for interview (but completed questionnaires)

44 analysed in intent-to-treat sample assessment

0 declined to participate further

44 analysed in intent-to-treat sample

Figure 1. CONSORT diagram of participants' progress through each phase of the study 
Table 1.

Diagnostic Outcome Measures at Each Assessment Point

NET

CLIN

WLC

Percent free of primary anxiety diagnosis

12-week assessment

Completer sample

$15 / 41(36.6 \%) \quad 13 / 40(32.5 \%) \quad 1 / 24(4.2 \%)$

Intent-to-treat sample

$15 / 44(34.1 \%) \quad 13 / 44(29.5 \%) \quad 1 / 27(3.7 \%)$

6-month follow-up

Completer sample

23/37 (62.2\%) 21/36 (58.3\%)

Intent-to-treat sample

24/44 (54.5\%) 22/44 (50\%)

12-month follow-up

Completer sample

29/37 (78.4\%) 29/36 (80.6\%)

Intent-to-treat sample

30/44 (68.2\%) 30/44 (68.2\%)

Percent free of any anxiety diagnosis

12-week assessment

Completer sample

$8 / 41(19.5 \%) \quad 9 / 40(22.5 \%) \quad 1 / 24(4.2 \%)$

Intent-to-treat sample

$8 / 44(18.2 \%) \quad 9 / 44(20.5 \%) \quad 1 / 27(3.7 \%)$

6-month follow-up

$\begin{array}{lll}\text { Completer sample } & \text { 20/37 (54.1\%) } & 18 / 36(50.0 \%) \\ \text { Intent-to-treat sample } & 20 / 44(45.5 \%) & 18 / 44(40.9 \%)\end{array}$

12-month follow-up

Completer sample

Intent-to-treat sample
23/37 (62.2\%) 26/36 (72.2\%)

24/44 (54.5\%) 26/44 (59.1\%)

Note. Completer sample included participants with data available at the 12-week assessment time point. For the intent-to-treat sample, missing values were replaced by value at previous time point (e.g. diagnosis was retained from the baseline value). 
Online CBT for Adolescent Anxiety

Table 2

Estimated Marginal Means and Standard Errors for Continuous Variables from Baseline to 12-week Assessment

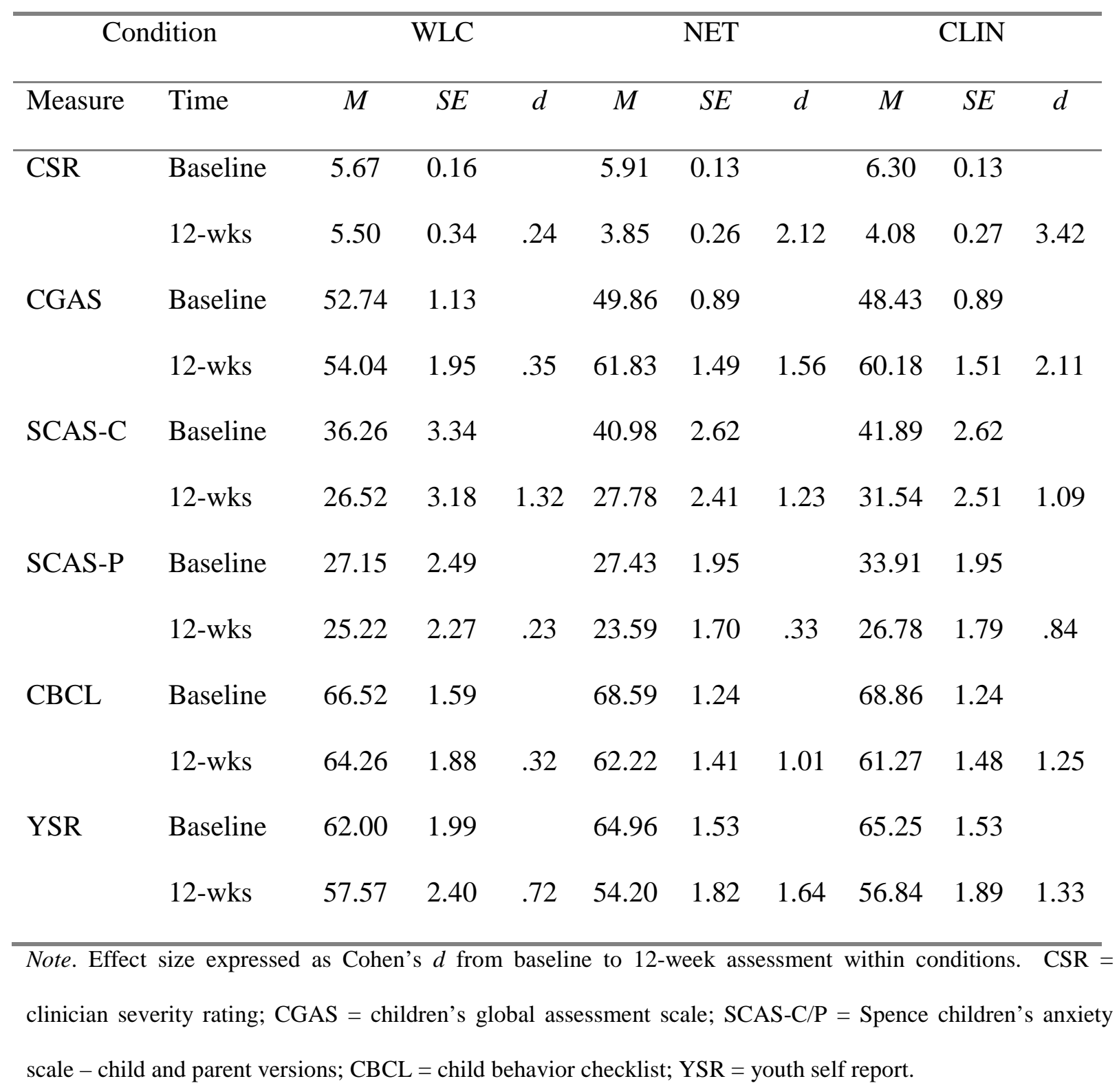




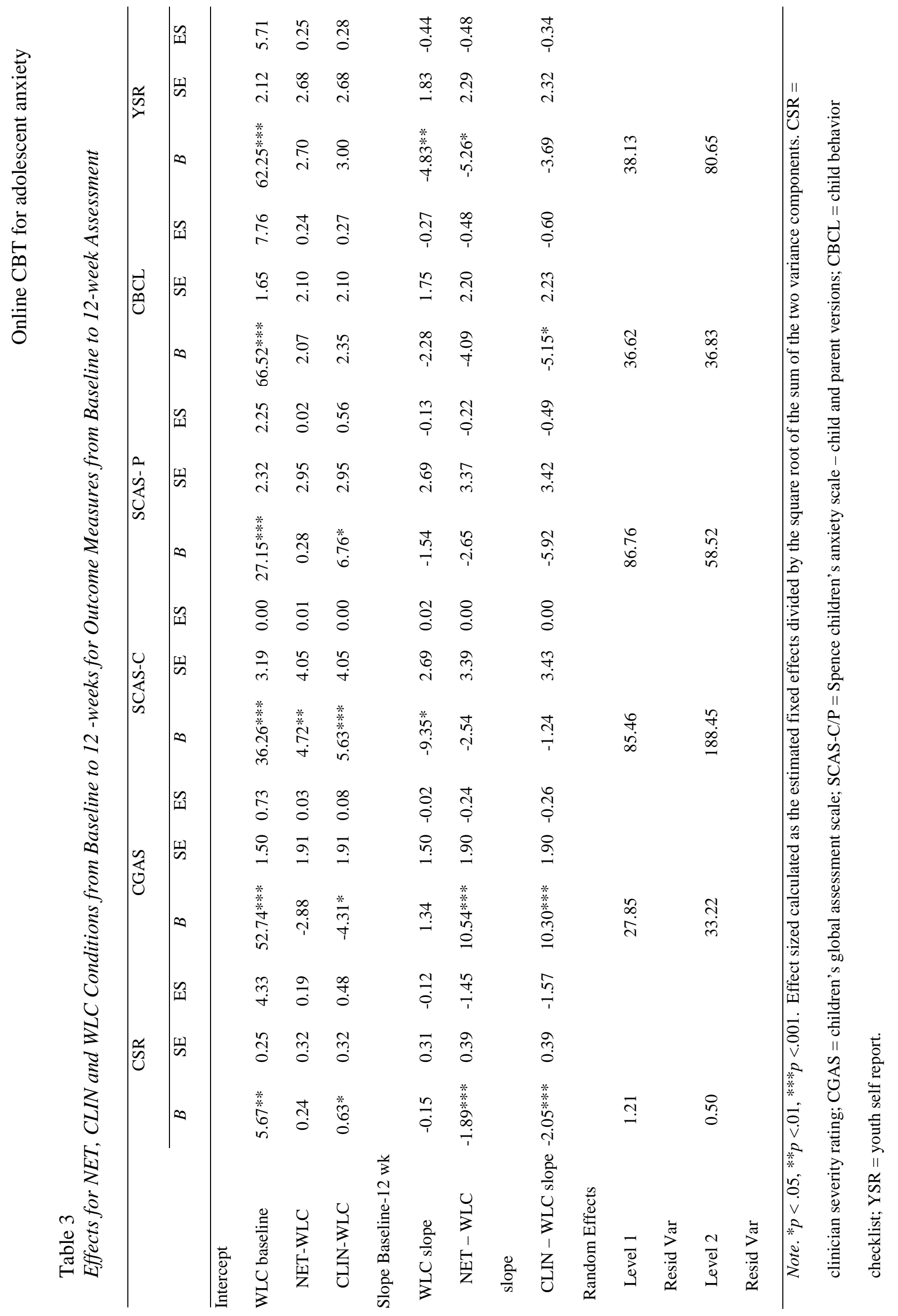


Table 4

Estimated Marginal Means and Standard Errors for Continuous Variables from Baseline to 12-week Assessment and 6- and 12-month Follow-ups for NET and CLIN Conditions

\begin{tabular}{|c|c|c|c|c|c|c|c|}
\hline & Condition & & ET & & & CLIN & \\
\hline Measure & Time & $M$ & $S E$ & $d$ & $M$ & $S E$ & $d$ \\
\hline \multirow[t]{4}{*}{ CSR } & Baseline & 5.91 & .12 & & 6.30 & .12 & \\
\hline & 12-wks & 3.85 & .29 & & 4.08 & .29 & \\
\hline & 6-mth fup & 2.60 & .32 & & 2.89 & .33 & \\
\hline & 12-mth fup & 1.97 & .34 & 2.58 & 1.81 & .35 & 3.88 \\
\hline \multirow[t]{4}{*}{ CGAS } & Baseline & 49.86 & .89 & & 48.43 & .89 & \\
\hline & 12-wks & 61.83 & 1.63 & & 60.18 & 1.65 & \\
\hline & 6-mth fup & 70.46 & 1.84 & & 68.19 & 1.87 & \\
\hline & 12-mth fup & 72.05 & 1.85 & 2.90 & 72.53 & 1.88 & 3.91 \\
\hline \multirow[t]{4}{*}{ SCAS-C } & Baseline & 40.98 & 2.62 & & 41.89 & 2.62 & \\
\hline & 12-wks & 27.78 & 2.48 & & 31.54 & 2.58 & \\
\hline & 6-mth fup & 22.52 & 2.59 & & 22.62 & 2.45 & \\
\hline & 12-mth fup & 20.20 & 2.42 & 1.85 & 17.94 & 2.49 & 2.08 \\
\hline \multirow[t]{4}{*}{ SCAS-P } & Baseline & 27.43 & 1.98 & & 33.91 & 1.98 & \\
\hline & 12-wks & 23.59 & 1.69 & & 26.78 & 1.77 & \\
\hline & 6-mth fup & 16.82 & 1.57 & & 17.53 & 1.51 & \\
\hline & 12-mth fup & 15.86 & 1.47 & 1.08 & 15.26 & 1.49 & 2.15 \\
\hline \multirow[t]{4}{*}{ CBCL } & Baseline & 68.59 & 1.33 & & 68.86 & 1.33 & \\
\hline & 12-wks & 62.22 & 1.53 & & 61.27 & 1.61 & \\
\hline & 6-mth fup & 58.88 & 1.80 & & 56.28 & 1.74 & \\
\hline & 12-mth fup & 55.39 & 2.01 & 1.76 & 55.89 & 2.11 & 1.80 \\
\hline \multirow[t]{4}{*}{ YSR } & Baseline & 64.96 & 1.54 & & 65.25 & 1.54 & \\
\hline & 12-wks & 54.20 & 1.88 & & 56.84 & 1.96 & \\
\hline & 6-mth fup & 51.94 & 2.01 & & 52.32 & 1.90 & \\
\hline & 12-mth fup & 49.29 & 2.24 & 1.67 & 49.91 & 2.31 & 1.65 \\
\hline
\end{tabular}

Note. Effect size expressed as Cohen's $d$ from baseline to 12-month assessment within conditions. CSR = clinician severity rating; CGAS = children's global assessment scale; SCAS-C/P = Spence children's anxiety scale - child and parent versions; CBCL = child behavior checklist; YSR = youth self report. 
苟

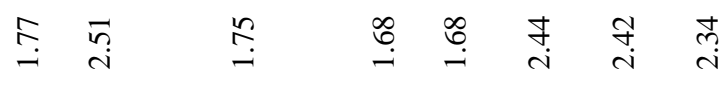

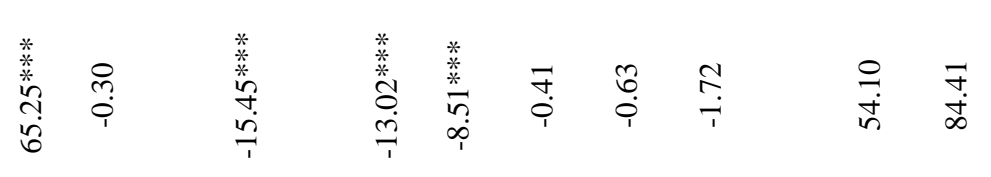

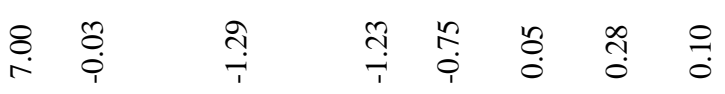

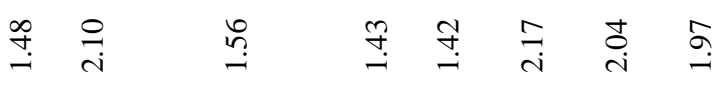

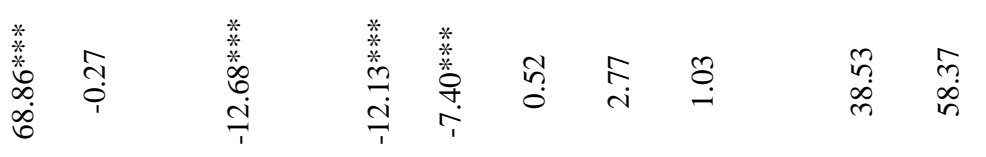

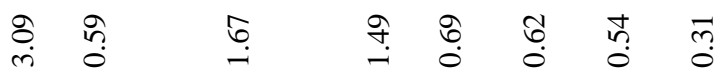

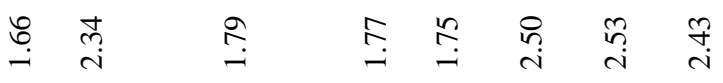

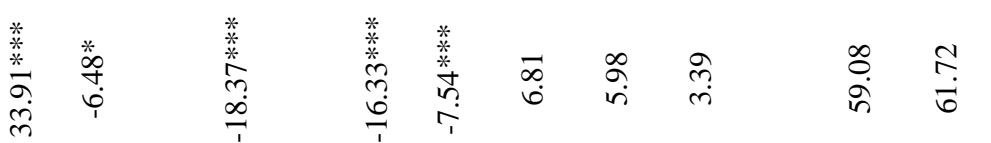

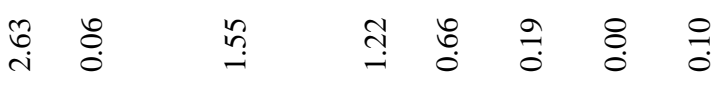

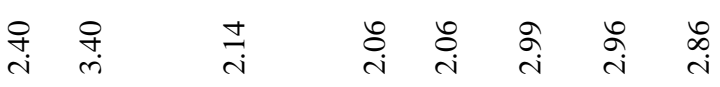

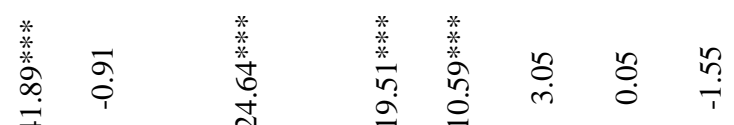

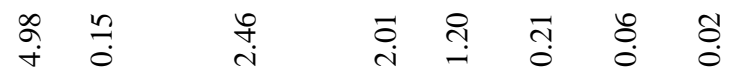

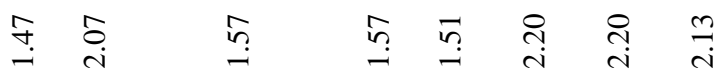

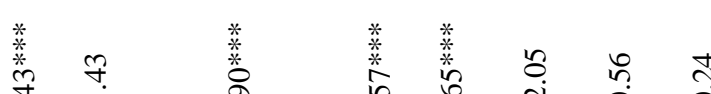

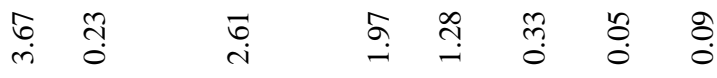

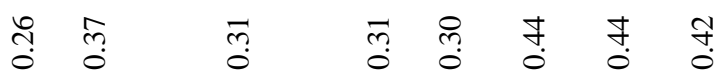

䕣

縠

尌

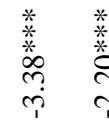

s.

동 : 영

$\stackrel{0}{0}$

兽

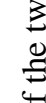

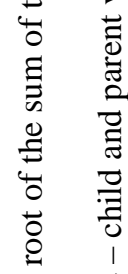

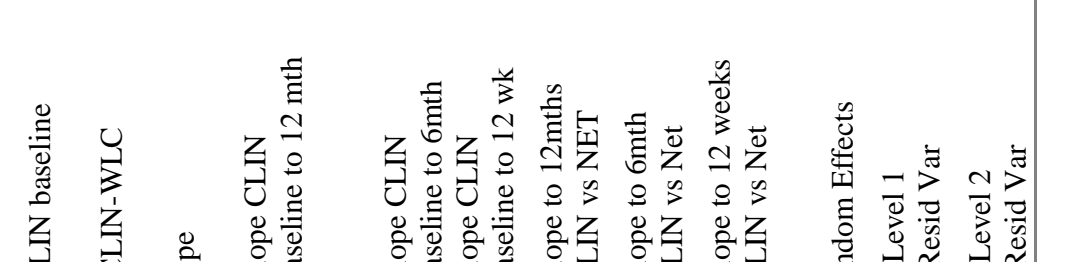
莺 


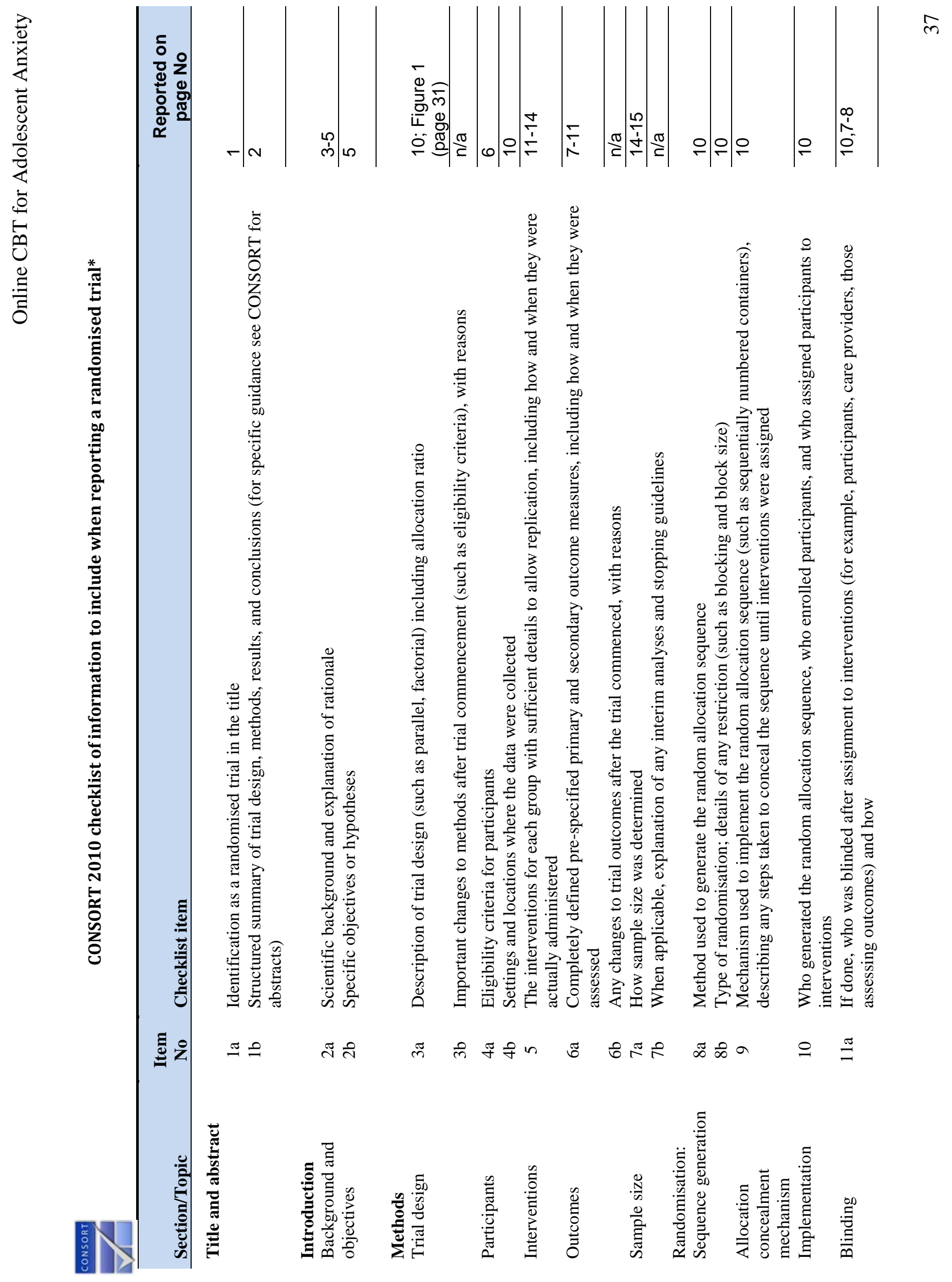




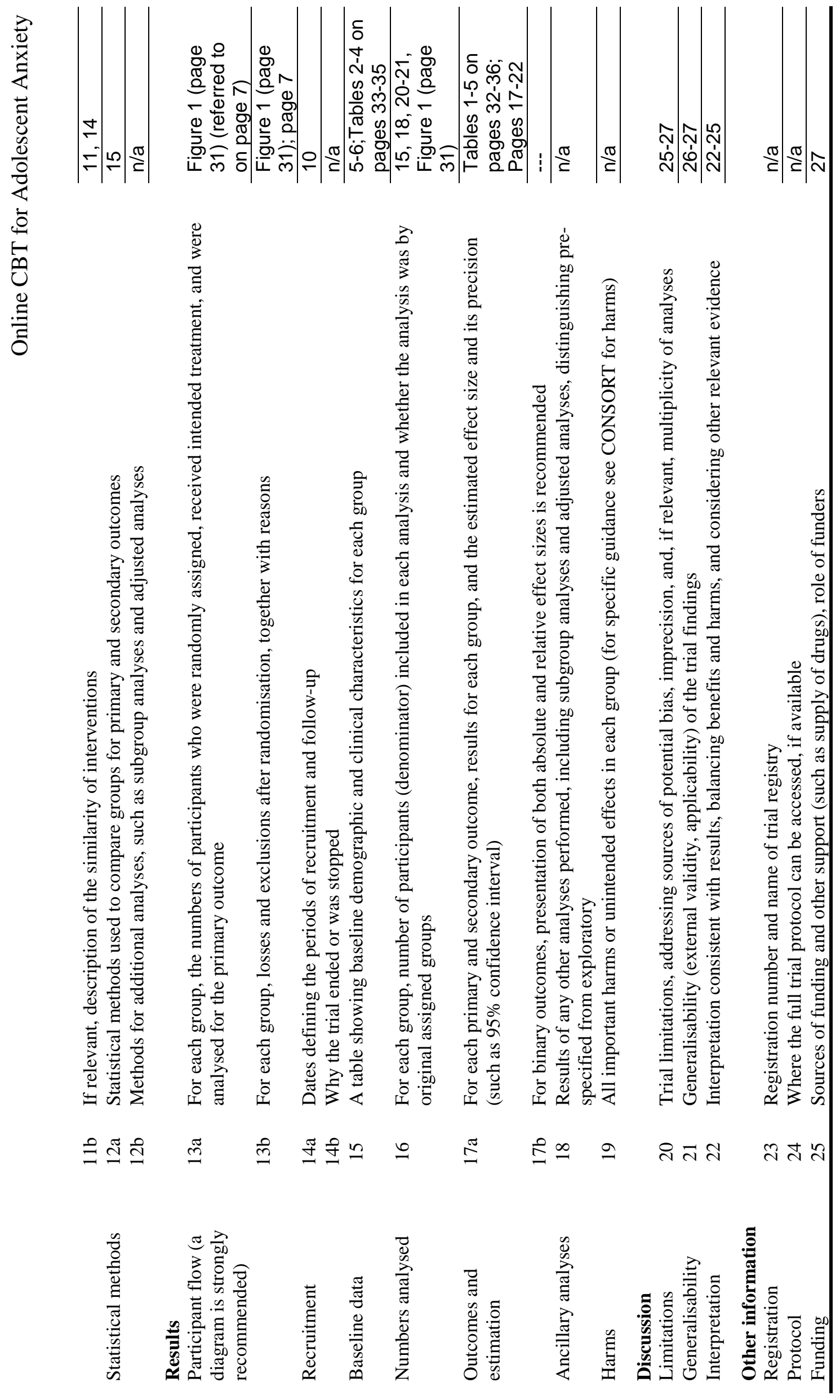

